\title{
Primary carnitine deficiency in two sisters with intractable epilepsy and reversible metabolic cardiomyopathy: Two case reports
}

\author{
XIU-FANG YANG ${ }^{1}$, GUO-SHENG LIU $^{2}$ and BING $\mathrm{YI}^{3}$ \\ ${ }^{1}$ Department of Pediatrics and Neonatology, Zhongshan Hospital Affiliated to Sun Yat-Sen University, \\ Zhongshan, Guangdong 528400; ${ }^{2}$ Department of Neonatology, The First Affiliated Hospital of Jinan University, \\ Guangzhou, Guangdong 510630; ${ }^{3}$ Molecular Inspection Center of Clinical Laboratory, Zhongshan Hospital \\ Affiliated to Sun Yat-Sen University, Zhongshan, Guangdong 528400, P.R. China
}

Received September 8, 2019; Accepted July 29, 2020

DOI: $10.3892 /$ etm.2020.9246

\begin{abstract}
Primary carnitine deficiency (PCD) is a disorder of the carnitine cycle that results in defective fatty acid oxidation. When carnitine cannot be transported into the cells, fatty acid oxidation is impaired, resulting a variety of symptoms, such as chronic muscle weakness, cardiomyopathy, hypoglycemia and liver dysfunction. The clinical manifestations and outcomes of different cases with PCD vary among patients. The present case report focused on two sisters with PCD. The younger sister presented with intractable epilepsy, and the older sister presented with reversible metabolic cardiomyopathy. Potential mutations in the SLC22A5 gene were investigated within the family, and a nonsense mutation [c.760C $>\mathrm{T}$ (p.R254X)] was identified in four family members. The two sisters harbored homozygous mutations, whereas their parents presented heterozygous mutations. Metabolic disease screening revealed low plasma free carnitine levels $(<5 \mu \mathrm{mol} / \mathrm{l})$ in the two sisters. The plasma free carnitine levels of their parents were normal, and they were asymptomatic. PCD in the two patients was managed using oral levocarnitine. The metabolic cardiomyopathy of the older sister improved following 3 months of treatment. However, the epilepsy of the younger sister was
\end{abstract}

Correspondence to: Dr Xiu-Fang Yang, Department of Pediatrics and Neonatology, Zhongshan Hospital Affiliated to Sun Yat-Sen University, 2 Sunwen Road, Zhongshan, Guangdong 528400, P.R. China

E-mail: 1104539484@qq.com

Dr Guo-Sheng Liu, Department of Neonatology, The First Affiliated Hospital of Jinan University, 613 Huangpu West Road, Guangzhou, Guangdong 510630, P.R. China

E-mail: tlgs@jnu.edu.cn

Abbreviations: PCD, primary carnitine deficiency; HPLC/MS/MS, high-performance liquid chromatography tandem mass spectrometry

Key words: PCD, encephalopathy, cardiomyopathy, gene mutation recurrent with oral antiepileptic therapy lasting one year and eight months, and epilepsy was finally controlled following right cerebral resection. The present case report demonstrated that the clinical manifestations presented by patients with PCD within the same family were different. The results indicated that treatment with levocarnitine supplementation should be initiated as soon as possible before irreversible organ damage occurs. In addition, metabolic decompensation and cardiac muscle functions were improved following carnitine supplementation. The resection of the severely diseased unilateral brain combined with carnitine supplementation and antiepileptic therapy may be an effective treatment for PCD with intractable epilepsy complications.

\section{Introduction}

Primary carnitine deficiency (PCD) is a fatal autosomal recessive disorder of fatty acid oxidation resulting from the defective transportation of carnitine into the kidney, heart and muscles (1). The SLC22A5 gene was originally identified in these tissues (1). PCD is typically characterized by episodes of hypoketotic hypoglycemia, hepatomegaly, elevated transaminases and hyperammonemia in infants; skeletal myopathy, high levels of creatine kinase and cardiomyopathy in childhood; or fatigability in adulthood (2-4).

\section{Case report}

A 15-month-old girl was admitted to Zhongshan Hospital Affiliated to Sun Yat-Sen University (Zhongshan, China) in November 2015 due to cough that had lasted for 3 days and a seizure, which lasted for $5 \mathrm{~h}$. She did not present wheezing, cyanosis, fever or vomiting and exhibited no notable symptoms. No abnormalities were noted at birth, with a birth weight of $3.1 \mathrm{~kg}$ and a body length of $50 \mathrm{~cm}$. The physical and motor development was normal. The patient exhibited recurrent forced clonic convulsions 4-5 times/day following admission and suffered from a coma with convulsions. No abnormalities were observed in the cardiopulmonary and abdominal physical examination, and physiological reflexes 
were present with Babinski sign. The white blood cell count in the peripheral blood was 16,160 cells $/ \mathrm{mm}^{3}$. The results of serum c-reactive protein, lactic acid, blood ammonia biochemistry and cerebrospinal fluid analyses were normal. The results of an electroencephalogram indicated large and slow waves originating from multiple right occipital areas and large paroxysmal wave rhythms in the left occipital area (Fig. 1; arrows). Echocardiography revealed a normal internal structure and heart functions. Brain MRI (November 2015) indicated a low signal in the right cerebral hemisphere on the T1-weighted image and a high signal on the T2-weighted image, which were primarily localized in the parietal occipital temporal lobe. The ventricular system was symmetrical with no enlargement, and the midline did not shift (Fig. 2). The disease management included treatment with ceftriaxone $(100 \mathrm{mg} / \mathrm{kg} / \mathrm{day})$, immunoglobulin ( $1 \mathrm{~g} / \mathrm{kg} /$ day for 2 days), methylprednisolone (2 mg/kg/day), mannitol (1 g/kg/day) and airway nursing, as well as a symptomatic treatment with oxcarbazepine (15 mg/kg; twice/day) as an antiepileptic drug. The screening for metabolic diseases revealed that plasma free carnitine level was low $(1.3 \mu \mathrm{mol} / 1$; normal range, $25-50 \mu \mathrm{mol} / 1)$, indicating that the patient suffered from PCD. The patient was treated with levocarnitine at a dose of $50-400 \mathrm{mg} / \mathrm{kg} / \mathrm{day}$ divided into three doses and a low-fat and medium-chain triglyceride-rich diet. The plasma free carnitine levels of the patient returned to normal levels after 1 month. Brain MRI (February 2016) indicated atrophy in the right cerebral hemisphere and an enlarged cortical lamellar necrosis (Fig. 3). The patient suffered from intractable epilepsy despite oral oxcarbazepine administration for 8 months. Subsequently, the antiepileptic therapy was altered to oral sodium valproate $(5 \mathrm{ml}$; twice/day) and oral levetiracetam (0.375 g; twice/day). Despite these treatments, the epileptic symptoms remained recurrent. In order to control the epilepsy, the patient was subjected to a right cerebral resection to excise the severely diseased lateral brain area in June 2017, following which the epilepsy was controlled. Brain MRI scans (August 2017) demonstrated alterations following right cerebral hemisphere resection and right cerebral basal ganglia resection, with compensatory hydrocephalus in the right frontotemporal occipital lobe and chronic hematoma and local crescent subdural effusion in the left frontal lobe (Fig. 4). The muscle strength and tension of the right limbs and the left upper limb of the patient were normal, but the muscle power of the left lower limb was grade 4 according to the grading system of muscle power (5), and the muscle tone was determined as paratonia of hypertonia (5). In addition, the language ability of the patient was normal.

The older sister of the proband, aged 3 years and 10 months, complained of malaise and fatigue that lasted for 1 week, and was admitted to Zhongshan Hospital Affiliated to Sun Yat-Sen University in February 2016. She did not present with cough, chest tightness, shortness of breath, cyanosis, vomiting, fever, diarrhea or convulsions. Her appetite and urine were normal. The physical and auxiliary examinations indicated that she exhibited PCD with cardiomyopathy complications, but without damage to the other organs. Echocardiography indicated a left atrial and left ventricular enlargement, as well as cardiac insufficiency. The left atrial diameter was $24 \mathrm{~mm}$, and the left ventricular diameter was $40 \mathrm{~mm}$. In addition, the left ventricular ejection fraction was $48 \%$. Brain MRI presented no abnormalities. The blood amino acid and acylcarnitine spectrum analysis of inherited metabolic diseases indicated that free carnitine $(0.66 \mu \mathrm{mol} / 1$; normal range, $25-50 \mu \mathrm{mol} / \mathrm{l})$ and a variety of acylcarnitine compounds (e.g. succinylcarnitine, butyrylcarnitine and propionylcarnitine, among others) were low, suggesting PCD. She was immediately treated with levocarnitine (100 mg/kg daily) and a low-fat and rich-medium-chain triglyceride diet, and the symptoms improved after 3 months. The size and function of her heart were restored to normal with the left atrial diameter of $21 \mathrm{~mm}$, the left ventricular diameter of $35 \mathrm{~mm}$, the left ventricular ejection fraction of $75 \%$ and normal physical development following 3 months of therapy.

Four members of the family were screened for congenital genetic metabolic diseases and SLC22A5 gene mutations using high-performance (HP) liquid chromatography (LC) tandem mass spectrometry (HPLC/MS/MS) and Sanger sequencing. For the HPLC, the following systems and conditions were used: HPLC system (LC20ADXR; Shimadzu Corporation); column, Agilent Zorbax SB-C18 $3.5 \mu \mathrm{m}, 2.1 \times 100 \mathrm{~mm}$ (Agilent Technologies, Inc.); environment temperature, $25 \pm 2^{\circ} \mathrm{C}$; environment humidity, 35-80\%; sample quantity (15 $\mu \mathrm{l})$; composition of mobile phase, one solvent of formic-and-methanol solution from NeoBase Non-derivatized MSMS kit (PerkinElmer, Inc.); and flow rate, $0.235 \mathrm{ml} / \mathrm{min}$ for sample injection before $0.20 \mathrm{~min}$; $0.017 \mathrm{ml} / \mathrm{min}$ for ionization from 0.21 to $1.10 \mathrm{~min}$; $0.7 \mathrm{ml} / \mathrm{min}$ for flushing the pipe from 1.15 to $1.45 \mathrm{~min}$; and $0.235 \mathrm{ml} / \mathrm{min}$ for equilibrium to the end. For the LC/MS/MS, in order to improve the sensitivity, the analytical measurements were performed in the multiple reaction monitoring mode (MRM). The MRM parameters were set according to the operating manual provided by Neobase Non-derivatized MSMS kit (MRM transition masses, CO $162.20 \mathrm{~m} / \mathrm{z}>103.10 \mathrm{~m} / \mathrm{z}$, CO IS $171.20 \mathrm{~m} / \mathrm{z}>103.10 \mathrm{~m} / \mathrm{z}$, C2 $241.10 \mathrm{~m} / \mathrm{z}>85.10 \mathrm{~m} / \mathrm{z}$, C2 IS $207.10 \mathrm{~m} / \mathrm{z}>85.10 \mathrm{~m} / \mathrm{z}$, C3 $218.10 \mathrm{~m} / \mathrm{z}>85.10 \mathrm{~m} / \mathrm{z}$, C3 IS $221.10 \mathrm{~m} / \mathrm{z}>85.10 \mathrm{~m} / \mathrm{z}$; Dwell time, $0.025 \mathrm{sec}$; collision energy, 14-18 V). The following systems and conditions were used: LC/MS/MS system (AB SCIEX API 3200 MD; AB Sciex LLC); ionization mode, positive; nitrogen gas temperature, $400^{\circ} \mathrm{C}$, nebuliser pressure, $30 \mathrm{psi}$; and flow rate, $0.235 \mathrm{ml} / \mathrm{min}$. For Sanger sequencing, after informed consent of the subjects' guardians, $2 \mathrm{ml}$ venous blood (with EDTA anticoagulation) were collected from the four members of the family. Genomic DNA was extracted via routine phenol chlorine method. The primers were designed with Primer Premier version 5.0 software (Premier Biosoft International) to amplify all exons and introns of the SLC22A5 gene. A PCR machine (C1000; Bio-Rad Laboratories, Inc.) was used to amplify the extracted DNA. The PCR reaction volume was $25 \mu 1$, including Takara La Taq premix (Takara Biotechnology Co., Ltd.) $12.5 \mu 1$, upstream and downstream primer mixture $0.75 \mu \mathrm{l}(10 \mathrm{pmol} / \mu \mathrm{l})$, genomic DNA $100 \mathrm{ng}$ and deionized water to $25 \mu \mathrm{l}$. The PCR reaction conditions were as follows: Pre-denaturation at $95^{\circ} \mathrm{C}$ for $3 \mathrm{~min}$; denaturation at $94^{\circ} \mathrm{C}$ for $30 \mathrm{sec}$, annealing at $60^{\circ} \mathrm{C}$ for $30 \mathrm{sec}$, extension for $40 \mathrm{sec}$ at $72^{\circ} \mathrm{C}$ for 38 cycles; and final extension for $8 \mathrm{~min}$ at $72^{\circ} \mathrm{C}$. The amplified PCR products were identified by $1.5 \%$ agarose gel electrophoresis (Bio-Rad Laboratories, Inc.), and the sequencing results were compared with the SLC22A5 gene sequences of the human genome in GenBank (http://www. ncbi.nlm.nih.gov/Genbank). One nonsense mutation [c.760C $>\mathrm{T}$ (p.R254X)] was identified. The two sisters exhibited homozygous 


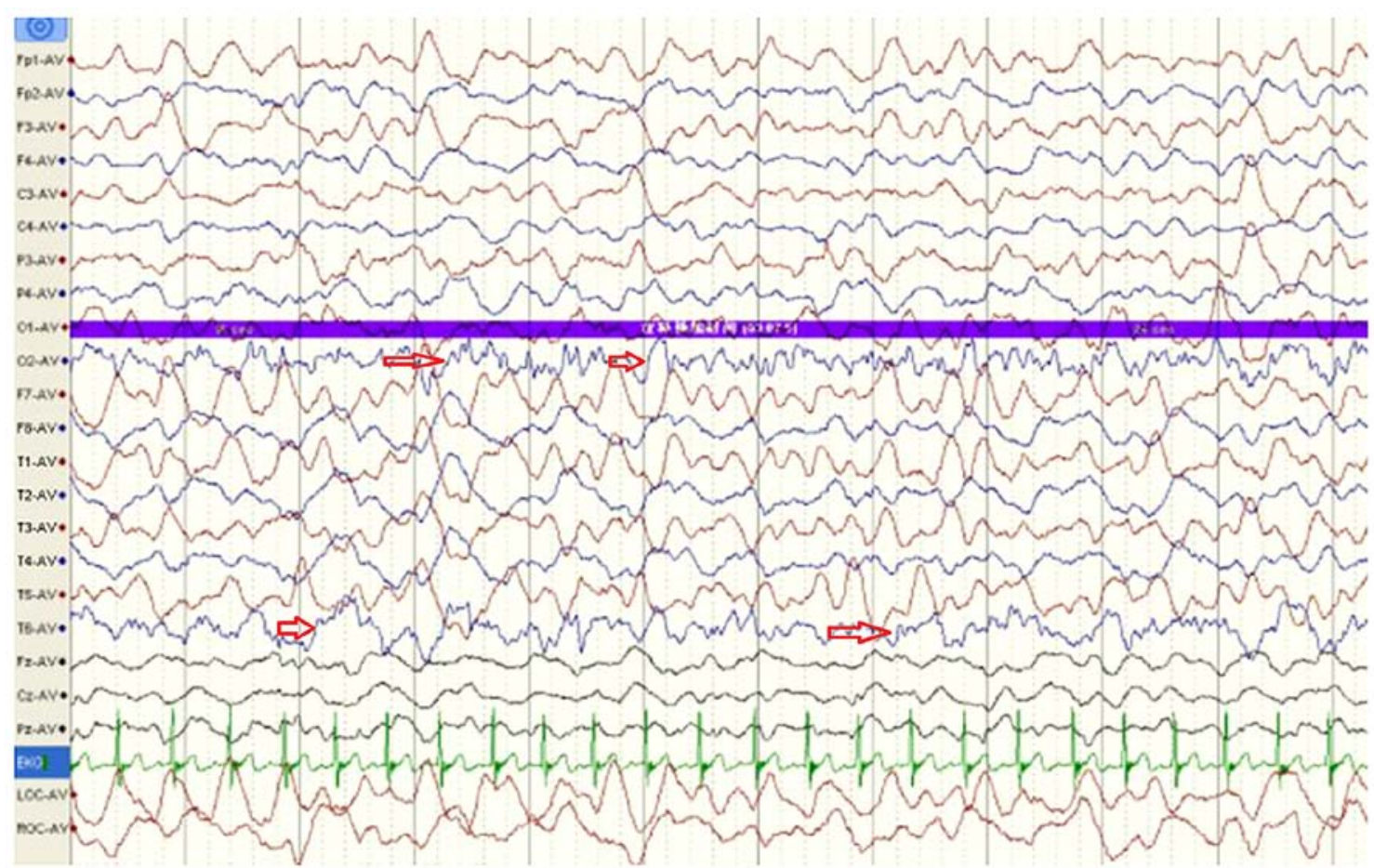

Figure 1. Electroencephalogram of the patient with primary carnitine deficiency and intractable epilepsy recorded in November 2015. The electroencephalogram indicated large and slow waves originating from multiple right occipital areas and large paroxysmal wave rhythms in the left occipital area, indicated by arrows.

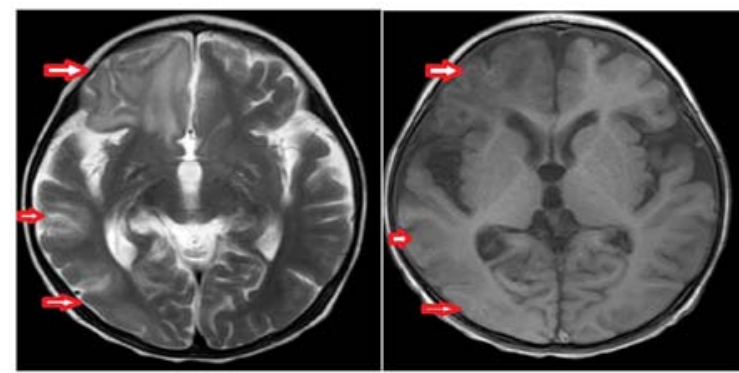

Figure 2. Brain MRI scans of the patient with primary carnitine deficiency and intractable epilepsy in November 2015. The brain MRI indicated a low signal in the right cerebral hemisphere on the T1-weighted image (right) and a high signal on the T2-weighted image (left), indicated by arrows.

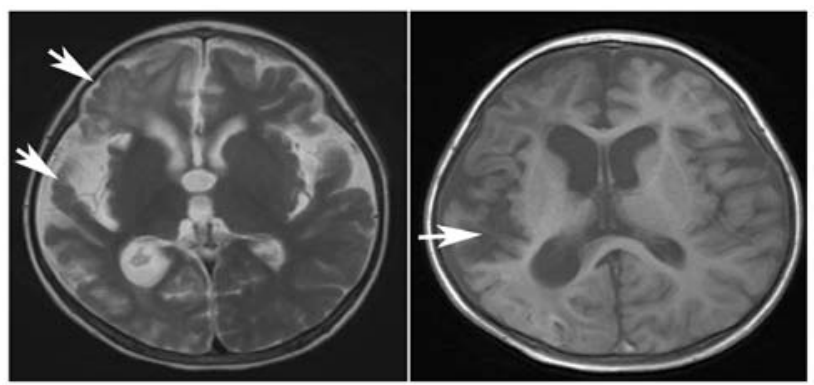

Figure 3. Brain MRI scans of the patient with primary carnitine deficiency and intractable epilepsy in February 2016. The brain MRI indicated atrophy in the right cerebral hemisphere and an enlarged cortical lamellar necrosis, low signal in the right cerebral hemisphere on the T1-weighted image (right) and high signal on the T2-weighted image (left), indicated by arrows.

mutations, whereas their parents exhibited heterozygous mutations (Fig. 5). The plasma free carnitine levels of the parents were

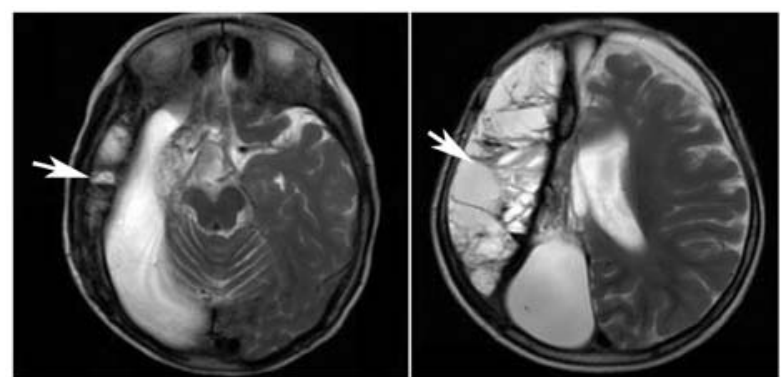

Figure 4. Brain MRI scans of the patient with primary carnitine deficiency and intractable epilepsy in August 2017. The brain MRI indicated a compensatory hydrocephalus in the right frontotemporal occipital lobe on the two different levels on the T2-weighted image (left and right image), indicated by arrows.

normal. Electrocardiogram, ultrasound, biochemical and brain MRI examinations of the family members were performed; the results demonstrated that all family members had not developed episodes of hypoketotic hypoglycemia, hepatomegaly, elevated transaminases or hyperammonemia. The parents were asymptomatic.

\section{Discussion}

PCD is an autosomal recessive disease caused by a functional defect in the carnitine transporter, which is encoded by the SLC22A5 gene (6). The incidence rate of PCD in Japan and Europe is $1: 40,000-1: 120,000(7,8)$. The principal function of levocarnitine is to assist in the transport of long chain fatty acids into the mitochondria to undergo $\beta$-oxidation (9). High-performance liquid chromatography tandem mass spectrometry (HPLC/MS/MS) is one of the methods that are used to test for metabolic disorders $(10,11)$; therefore, HPLC/MS/MS 


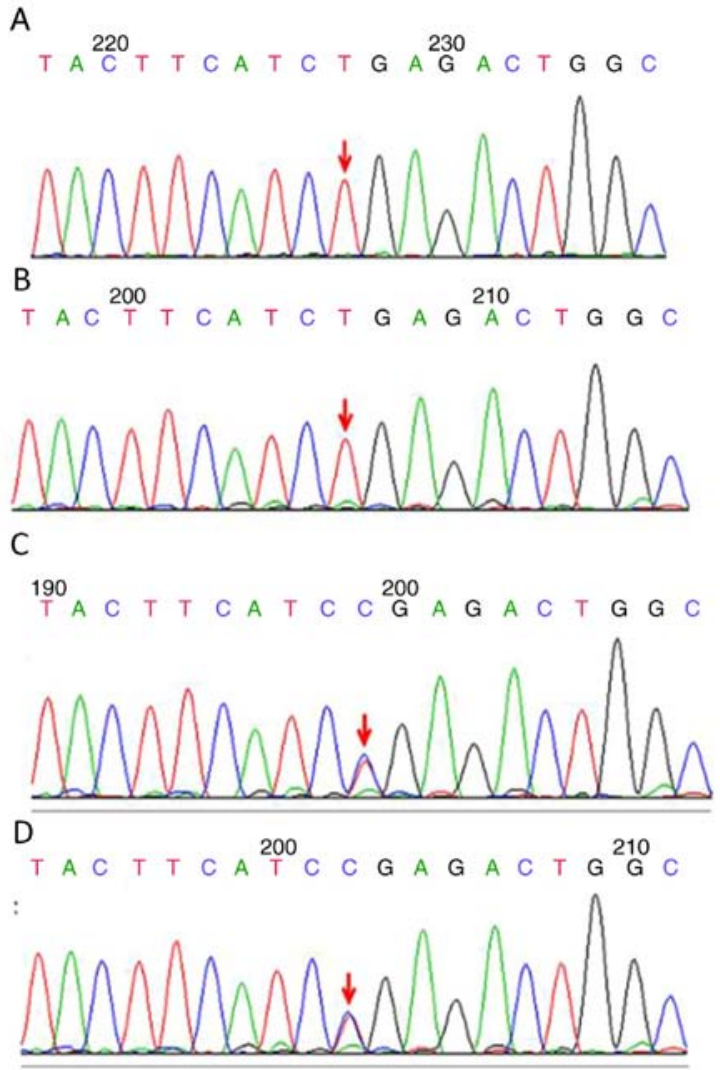

Figure 5. Nonsense homozygous mutation [c.760C $>\mathrm{T}$ (p.R254X)] in the SLC22A5 gene in the proband and her elder sister and heterozygous mutation in their parents. The arrows indicate the site of the mutation. (A), the proband; (B), the elder sister; (C), their mother; (D), their father.

screening was applied in the present case report. Carnitine deficiency has been indicated to result in a reduction of energy generation and the accumulation of long chain fatty acids in the cytoplasm, as well as a number of biochemical abnormalities (hypoglycemia, liver dysfunction, etc.) and organ damage $(12,13)$. The principal clinical manifestations include hypoketotic hypoglycemia, encephalopathy, cardiomyopathy and skeletal muscle weakness. The diagnoses of PCD in the present case report were based on the low levels of plasma free carnitine $(<5 \mu \mathrm{mol} / \mathrm{l})$. Lin et al (14) investigated the potential mutations in the SLC22A5 gene in patients with PCD, and the diagnosis of eight patients with PCD was confirmed at the gene level. A total of six mutations were identified in the SLC22A5 gene, including one novel mutation, which expanded the existing mutation spectrum of the SLC22A5 gene (14). In the general population, the incidence of heterozygous mutations ranges between 0.5 and $1 \%$, and most frequently occurs between 1 month and 7 years of age (7). The SLC22A 5 gene is localized in chromosome $5 \mathrm{q} 31$ and contains 10 exons (15). The reported mutations are localized in exons 1-9 and introns 3, 7 and 8 , and exonal mutations have been indicated to be the most frequent (16). The low levels of plasma free carnitine are an indirect effect of the dysregulation in glucose aerobic oxidation, gluconeogenesis and ketogenesis, as well as other metabolic pathways, which has been reported to result in metabolic disorders and organ damage (15). When carnitine concentrations in the plasma are $10-20 \%$ lower than normal levels, carnitine deficiencies may induce a number of clinical symptoms in the liver, heart, muscles and brain (15). In 1975, the first case if PCD was reported, since which the disease has received increasing clinical attention (17). Over the last decade, owing to the use of MS/MS analysis of dried blood spots, the acylcarnitine spectrum and improvements in the determination of amino acid chromatography and urine organic acid gas mass spectrometry for genetic metabolic disease detection, a greater number of children have received timely diagnosis $(18,19)$.

The therapeutic dose of levocarnitine should be adjusted according to alterations in its concentration in the blood of individual patients, as well as the blood alkalinity and the degree of the disease in order to maintain the stability of the tissues and blood. A high dose levocarnitine treatment may cause diarrhea, nausea and other gastrointestinal discomforts; however, the dose can be reduced and once the adverse reactions have improved, it can be gradually increased to the initial treatment dose (20). Patients with PCD require a lifelong treatment with levocarnitine, and a sudden withdrawal may result in a rapid drop in the plasma carnitine concentration, causing recurrent Reye syndrome and even sudden death (21). For asymptomatic patients with PCD, treatment with levocarnitine has been indicated to be effective in preventing morbidity and sudden death (22). In the present case report, although the two sisters with PCD exhibited a homozygous mutation in SLC22A5 [c.760 C > T (p.R254X)], their clinical manifestations varied. Following treatment of the proband with levocarnitine and internal medical therapies (including antiepileptic therapy and rehabilitation), the serum free carnitine levels were restored to normal levels, but the clinical symptoms recovered slowly, and sequelae in the nervous system remained. A 3-year pilot study in the Zhejiang province in China has suggested that mental retardation and motor delay were difficult to reverse in the majority of symptomatic patients with PCD following a late diagnosis (23). In the present study, although the older sister of the proband exhibited cardiac complications, following oral administration of levocarnitine, the clinical symptoms improved rapidly. It has been reported that oral carnitine supplementation in infants with PCD-related cardiomyopathy result in improved clinical outcomes with the ejection fraction reaching $75 \%$ following 3-month treatment $(24,25)$. Previous reports have also suggested that excising the severely diseased lateral brain may be used to treat refractory epilepsy, with epilepsy and functional outcomes being improved following surgery (26-28), which was in agreement with the treatment results observed in the proband in the present case report.

In conclusion, the two cases and the pedigree analysis revealed that clinical manifestations of PCD within the same family were specific to each individual. The results also demonstrated that treatment with levocarnitine supplementation should be initiated as early as possible before irreversible organ damage occurs. In addition, metabolic decompensation and cardiac muscle functions were demonstrated to improve after carnitine supplementation. The resection of the severely diseased unilateral brain combined with carnitine supplementation and antiepileptic therapy may be an effective treatment strategy for PCD with intractable epilepsy.

\section{Acknowledgements}

Not applicable. 


\section{Funding}

No funding was received.

\section{Availability of data and materials}

The datasets used and/or analyzed during the current study are available from the corresponding author on reasonable request.

\section{Authors' contributions}

XFY conceived the study, participated in its design and coordination and drafted the manuscript. GSL performed the clinical diagnosis and treatment of the patients. BY performed the molecular genetic studies, participated in the sequence alignment and drafted the manuscript. All authors read and approved the final manuscript.

\section{Ethics approval and consent to participate}

Informed consent for participation was received from the patients' guardians, and the protocol was approved by the Ethical Committee of Zhongshan Hospital Affiliated to Sun Yat-Sen University, Zhongshan, China (approval no. B2015111601).

\section{Patient consent for publication}

Informed consent was obtained from the guardians of the two patients.

\section{Competing interests}

The authors declare that they have no competing interests.

\section{References}

1. Tan JQ, Chen DY, Li ZT, Yan TZ, Huang JW and Cai R: Genetic diagnosis of 10 neonates with primary carnitine deficiency. Zhongguo Dang Dai Er Ke Za Zhi 19: 1150-1154, 2017 (In Chinese).

2. Buist NR: Historical perspective on clinical trials of carnitine in children and adults. Ann Nutr Metab 68: 1-4, 2016.

3. Ravindranath A, Pai G, Srivastava A, Poddar U and Yachha SK Infant with hepatomegaly and hypoglycemia: A setting for fatty acid oxidation defects. Indian J Gastroenterol 36: 429-434, 2017.

4. Belousova ED: The decreased level of plasma carnitine in patients with epilepsy. Zh Nevrol Psikhiatr Im S S Korsakova 117: 106-110, 2017 (In Russian).

5. Greenberg DA, Aminoff MJ and Simon RP: Clinical Neurogy (5th edition). The United States: Mc Graw-Hill, New York, NY, pp157-158, 2002.

6. Ferdinandusse S, Brinke HT, Ruiter JP, Haasjes J, Oostheim W, Ven Lenthe H, IJlst L, Ebberink MS, Wanders RJ, Vaz FM and Waterham HR: Mutation creating an upstream translation initiation codon in SLC22A5 5'UTR is a frequent cause of primary carnitine deficiency. Hum Mutat 40: 1899-1904, 2019.

7. Koizumi A, Nozaki J, Ohura T, Kayo T, Wada Y, Nezu J, Ohashi R, Tamai I, Shoji Y, Takada G, et al: Genetic epidemiology of the carnitine transporter OCTN2 gene in a Japanese population and phenotypic characterization in Japanese pedigrees with primary systemic carnitine deficiency. Hum Mol Genet 8: 2247-2254, 1999.

8. Hassan FA, El Mougy F, Sharaf SA, Mandour I, Morgan MF, Selim LA, Hassan SA, Salem F, Oraby A, Girgis MY, et al: Inborn errors of metabolism detectable by tandem mass spectrometry in Egypt: The first newborn screening pilot study. J Med Screen 23 $124-129,2016$
9. Nałęcz KA and Nałęcz MJ: Carnitine-mitochondria and beyond. Postepy Biochem 62: 85-93, 2016.

10. Lindner M, Gramer G, Haege G, Fang-Hoffmann J, Schwab KO, Tacke U, Trefz FK, Mengel E, Wendel U, Leichsenring M, et al: Efficacy and outcome of expanded newborn screening for metabolic diseases-report of 10 years from South-West Germany. Orphanet J Rare Dis 6: 44, 2011.

11. Lehotay DC, Hall P, Lepage J, Eichhorst JC, Etter ML and Greenberg CR: LC-MS/MS progress in newborn screening. Clin Biochem 44: 21-31, 2011.

12. Longo N, Frigeni M and Pasquali M: Carntiin transport and fatty acid oxidation. Biochim Biophys Acta 1863: 2422-2435, 2016.

13. Gallant NM, Leydiker K, Wilnai Y, Lee C, Lorey F, Feuchtbaum L, Tang H, Carter J, Enns GM, Packman S, et al: Biochemical characteristics of newborns with carnitine transporter defect identified by newborn screening in California. Mol Genet Metab 122: 76-84, 2017.

14. Lin Y, Lin W, Yu K, Zheng F, Zheng Z and Fu Q: Mutational analysis of SLC22A5 gene in eight patients with systemic primary carnitine deficiency. Zhonghua Yi Xue Yi Chuan Xue Za Zhi 34: 35-39, 2017 (In Chinese).

15. Frigeni M, Balakrishnan B, Yin X, Calderon FR, Mao R, Pasquali $\mathrm{M}$ and Longo N: Functional and molecular studies in primary carnitine deficiency. Hum Mutat 38: 1684-1699, 2017.

16. Li FY, El-Hattab AW, Bawle EV, Boles RG, Schmitt ES, Scaglia F and Wong LJ: Molecular spectrum of SLC22A5 (OCTN2) gene mutations detected in 143 subjects evaluated for systemic carnitine defciency. Hum Mutat 31: E1632-E1651, 2010.

17. Angelini C: Letter: Carnitine Deficiency. Lancet 2: 554, 1975.

18. Longo N: Primary carnitine deficiency and newborn screening for disorders of the carnitinecycle. Ann Nutr Metab 68: 5-9, 2016.

19. Zheng J, Zhang Y, Hong F, Yang J, Tong F, Mao H, Huang X, Zhou X, Yang R, Zhao Z and Huang X: Screening for fatty acid oxidation disorders of newborns in Zhejiang province: prevalence, outcome and follow-up. Zhejiang Da Xue Xue Bao Yi Xue Ban 46: 248-255, 2017 (In Chinese).

20. Deswal S, Bijarnia-Mahay S, Manocha V, Hara K, Shigematsu Y, Saxena R and Verma IC: Primary carnitine deficiency-a rare treatable cause of cardiomyopathy and massive hepatomegaly. Indian J Pediatr 84: 83-85, 2017.

21. Lahrouchi N, Lodder EM, Mansouri M, Tadros R, Zniber L, Adadi N, Clur SA, van Spaendonck-Zwarts KY, Postma AV, Sefiani A, et al: Exome sequencing identifies primary carnitine deficiency in a family with cardiomyopathy and sudden death. Eur J Hum Genet 25: 783-787, 2017.

22. Madsen KL, Preisler N, Rasmussen J, Hedermann G, Olesen JH, Lund AM and Vissing J: L-Carnitine improves skeletal muscle fat oxidation in primary carnitine deficiency. J Clin Endocrinol Metab 103: 4580-4588, 2018.

23. Jun JS, Lee EJ, Park HD and Kim HS: Systemic primary carnitine deficiency with hypoglycemic encephalopathy. Ann Pediatr Endocrinol Metab 21: 226-229, 2016.

24. Perin F, Rodríguez-Vázquez Del Rey MD, Carreras-Blesa C, Arrabal-Fernández L, Jiménez-Jáimez J and Tercedor L: Dilated cardiomyopathy with short QT interval suggests primary carnitine deficiency. Rev Esp Cardiol (Engl Ed) 71: 1074-1075, 2018.

25. Papadopoulou-Legbelou K, Gogou M, Dokousli V, Eboriadou M and Evangeliou A: Dilated cardiomyopathy as the only clinical manifestation of carnitine transporter deficiency. Indian J Pediatr 84: 231-233, 2017.

26. Morrison-Levy N, Go C, Ochi A, Otsubo H, Drake J, Rutka J and Weiss SK: Children with autism spectrum disorders and drug-resistant epilepsy can benefit from epilepsy surgery. Epilepsy Behav 85: 200-204, 2018.

27. Hirsch E and Arzimanoglou A: Children with drug-resistant partial epilepsy: Criteria for the identification of surgical candidates. Rev Neurol (Paris) 160: 5S210-5S219, 2004 (In French).

28. Roth J, Nagar S, Constantini S and Fried I: Hemispherotomy for treatment of refractory epilepsy in children. Harefuah 156: 482-485, 2017 (In Hebrew).

This work is licensed under a Creative Commons Attribution-NonCommercial-NoDerivatives 4.0 International (CC BY-NC-ND 4.0) License. 